\title{
Corrigendum
}

\section{An epidemiological survey of children's iodine nutrition and goitre status in regions with mildly excessive iodine in drinking water in Hebei Province, China - Corrigendum}

Shengmin $\mathrm{Lv}^{1}$, Jun Zhao ${ }^{1}$, Dong $\mathrm{Xu}^{1}$, Zhengshui Chong ${ }^{1}$, Lihui Jia', Yonggui Du', Jing $\mathrm{Ma}^{1}$ and Shannon Rutherford ${ }^{2}$

${ }^{1}$ Heibei Provincial Center for Disease Control and Prevention, No. 97, Huai'an East Road, Shijiazhuang 050021 , People's Republic of China: ${ }^{2}$ Centre for Environment and Population Health, School of Environment, Griffith University, Brisbane, Australia

First published online 18 February 2013

doi:10.1017/S1368980012000146, Published by Cambridge University Press, Public Health Nutrition volume 15, issue 7, pp. $1168-1173$

There are two errors as follows:

The author name Zhengshui Chong

should appear as Zhenshui Chong.

The author affiliation Heibei Provincial Center for Disease Control and Prevention

should appear as Hebei Province Center for Disease Prevention and Control, No. 97 Huai'an Donglu, Shijiazhuang 050021, Hebei Province, PR China

\section{Reference}

Lv S, Zhao J, Xu D, Chong Z, Jia L, Du Y, Ma J \& Rutherford S (2012) An epidemiological survey of children's iodine nutrition and goitre status in regions with mildly excessive iodine in drinking water in Hebei Province, China. Public Health Nutrition 15, 1168-1173. 